\title{
Development of Medium Pressure Laser Ionization, MPLI. Description of the MPLI Ion Source
}

\author{
Matthew F. Appel \\ Department of Biology, Chemistry, and Environmental Science, Christopher Newport University, \\ Newport News, Virginia, USA
}

\author{
Luke C. Short and Thorsten Benter \\ Bergische Universität Wuppertal, Wuppertal, Germany
}

A novel pulsed valve/ion source combination capable of time-resolved sampling from atmospheric pressure has been developed for use with laser ionization time of flight mass spectrometry. The source allows ionization extremely close to the nozzle of the pulsed valve, enabling ultra-sensitive detection of a number of compounds, e.g., NO, at mixing ratios $<1 \mathrm{pptV}$. Furthermore, at analyte mixing ratios in the $\mathrm{ppbV}$ range, the temporal resolution of the system is in the sub-second regime, allowing time-resolved monitoring of highly dynamic and complex mixtures, e.g., human breath or reacting chemical mixtures in atmospheric smog chamber experiments. Rotational temperatures of $\sim 50 \mathrm{~K}$ have been observed for analytes seeded in the supersonic jet expansion at a distance of $1 \mathrm{~mm}$ downstream of the nozzle orifice. The refinement of the original ion source has drastically reduced the impact of reflected laser light and the resultant electron impact signals previously observed. The general applicability of this technique is demonstrated here by coupling the source to commercially available as well as home-built time-of-flight mass spectrometers. Finally, we discuss the MPLI technique in view of the very recently introduced atmospheric pressure laser ionization (APLI) as well as the traditional jet-REMPI approach. (J Am Soc Mass Spectrom 2004, 15, 1885-1896) (C) 2004 American Society for Mass Spectrometry

$\mathrm{T}$ The time-resoled measurement of trace components in complex matrices is an ongoing challenge in the natural sciences. It has long been recognized that these trace species significantly influence the properties, chemical and dynamic behavior, impact, and efficacy of the host matrix, which can be solid, liquid, or gaseous. Examples for the latter are ambient air, vehicle exhaust, and human breath. As an illustration, an adult breathes approximately 10,000$12,000 \mathrm{~L}$ of air per day. Within that volume, there exists a variety of air pollutants, including particulate matter, volatile organic compounds, $\mathrm{NO}_{\mathrm{x}}$, and ozone. Although only present in trace (i.e., mixing ratios $<1 \mathrm{ppmV}$ ) amounts, these compounds are related to a series of health effects that include reduction in normal breathing, fatigue and confusion, inflammation of bronchial airways, and sore throat and headache [1].

In order to understand and eventually control the effects of these trace contaminants, a detailed picture of their sources, sinks, mixing ratios, impact, and role

Published online November 11, 2004

Address reprint requests to Dr. T. Benter, Bergische Universität Wuppertal, FB C, Chemie, Gauss Strasse 20, 42097 Wuppertal, Germany. E-mail: tbenter@uni-wuppertal.de in often highly dynamic processes is required. This can only occur if measurement techniques exist which allow time-resolved, selective detection at the trace and/or ultra-trace level. Towards this end, research in our laboratories has focused on the development of efficient ion generation schemes based on resonance enhanced multi-photon ionization (REMPI) for the detection of trace gases in reacting chemical systems, e.g., atmospheric smog chambers, ambient air, and human breath. Utilizing a novel pulsed valve capable of time-resolved sampling at atmospheric pressure coupled to a variety of time-of-flight (TOF) mass spectrometers, several atmospherically relevant trace gases have been selectively and sensitively detected in the laboratory. This technique was recently introduced by our group as "Atmospheric Pressure Laser Ionization Mass Spectrometry" (APLI-MS) [2, 3].

In view of a newly evolving laser ionization technique operating at ion source pressures around $\mathrm{p}=1 \mathrm{~atm}$ [4], the acronym APLI for the technique presented in this and previous papers becomes ambiguous. In order to describe the present technique more accurately, the term medium pressure laser ionization (MPLI) is used with the intention to better distinguish the three pressure regimes in which cur- 
rently applied laser ionization techniques operate: (1) The jet-REMPI approach operates in a low pressure background and the ionization region is located downstream of the sudden-freeze surface [5] of a gas jet. (2) As described in this article, the ionization region when using MPLI is located very close to the nozzle orifice and thus within the continuous region of the jet, approaching local pressures up-to 10 mbar. (3) When performing APLI, ionization occurs at atmospheric pressure, upstream of the mass spectrometer sampling orifice. We discuss the advantages and limitations of each technique in more detail.

The primary concern when developing MPLI was to establish detection limits in the relevant $\mathrm{ppbV}$ and sub-ppbV range, while simultaneously providing the ability to sample directly from ambient pressure. With this capacity, true temporal resolution on the second or even sub-second scale is possible, allowing monitoring of trace gases in highly dynamic matrices, e.g., reacting chemical systems in smog chamber experiments. In most of the current techniques that couple pulsed inlet systems with TOF analyzers, a supersonic jet is expanded into a differentially pumped region, as schematically shown in Figure 1a. After skimming the jet, molecules in the collimated molecular beam are ionized in a collision-free, high vacuum environment. Depending on the individual experimental set-up, the expansion is oriented perpendicular to or collinear with the ion optical axis and the distance from the nozzle to the ionization region is generally greater than $60 \mathrm{~mm}$ [6]. The main advantage of this configuration is the significant rotational cooling of the seeded molecules to a small number of molecular ground states. Nonetheless, due to the $1 / \mathrm{r}^{2}$ fall-off in molecule density within the expansion direction [7], these techniques sample the jet at relatively low analyte densities, severely limiting the maximum attainable sensitivity.

In order to increase the sensitivity associated with jet expansions, a number of attempts have been made to move the ionization region closer to the nozzle orifice. The majority of these systems introduce the expanding jet perpendicular to the ion flight tube axis as shown in Figure $1 \mathrm{~b}$ [8-11]. The jet-expansion is sampled anywhere between 15-25 mm downstream of the pulsed valve, just behind the sudden-freeze surface of the jet expansion, where the molecule density is still relatively high. Further reduction in the distance to ionization has been documented by Onoda et al. [12]. In this arrangement, the jet is expanded collinear with the axis of ion flight, shown in Figure 1c. Ionization occurs $10 \mathrm{~mm}$ downstream from the nozzle, roughly halfway between a repellor electrode and extraction grid.

The principle of operation of photo ionization techniques at atmospheric pressure, i.e., atmospheric pressure photo-ionization (APPI) and atmospheric pressure laser ionization (APLI) are schematically shown in Figure 1e. Ionization occurs at $\mathrm{p}=1 \mathrm{~atm}$, upstream of the sampling orifice or skimmer of the differential pumping stage. In this pressure region, the impact of ion-molecule reactions, relative ion mobilities, and gas flows through the sampling nozzle on the efficiency of such an ion source require careful attention.

In this contribution we report on the development and design of a novel pulsed TOF-MS inlet system for laser ionization ultimately close to the nozzle orifice, i.e., in the continuous, high-pressure region of the jet as shown in Figure 1d. We have quantitatively investigated the performance of this source with respect to cooling efficiency and sensitivity.

\section{Experimental}

\section{Mass Analyzers}

All stationary experiments were carried out using home-built differential pumping stages coupled to time-of-flight mass spectrometers. The first instrument, a Bruker TOF1 (Bremen, Germany), features a differential pumping stage equipped with a $450 \mathrm{ls}^{-1}$ turbo molecular pump (Alcatel, ATP5400, Wertheim, Germany) backed by a $16 \mathrm{~m}^{3} \mathrm{hr}^{-1}$ two-stage roughing pump (Balzers Duo 016, Balzers, Asslar, Germany), three fused silica laser windows, and high-voltage feed-throughs. In addition, a relatively small front plate-skimmer distance of $10 \mathrm{~cm}$ allows for flexible inlet stage adaptation. The TOF1 instrument is factory equipped with a combined electron impact/ photoionization source, a dual stage gridless reflectron, a cascaded multichannel plate detector, and fast control and data acquisition electronics (Bruker B500 digitizer/Motorola MVME 68060 based VME bus computer combination).

The second mass spectrometer employed was a Bruker TurboTOF instrument, also equipped with a home-built differential pumping stage, as described above. The pumping system consisted of a Balzers TMU-260 turbo pump backed by a Balzers Duo 5 roughing pump. The TurboTOF was of linear configuration and used identical data acquisition electronics, as described above.

The third system was a home-built mobile TOF instrument equipped with a single stage reflectron. The $50 \times 20 \times 20(\mathrm{~L} \times \mathrm{W} \times \mathrm{H}) \mathrm{cm}^{3}$ body was constructed as one piece from aluminum to be dimensionally compatible with a Pfeiffer TC 600 (Pfeiffer, Asslar, Germany) three-port turbo drag pump backed by a Pfeiffer Duo 10 roughing pump. The two high vacuum ports of the pump were directly connected to the differential pumping chamber and analyzer region of the main housing. In the present experiments, both chambers were separated by a $1 \mathrm{~mm}$ i.d. orifice, which could be easily replaced to match various operating pressure conditions. The mass spectrometer housing was equipped with vacuum ports for pressure monitoring as well as with three fused silica laser windows and various electrical feed-throughs. 
a
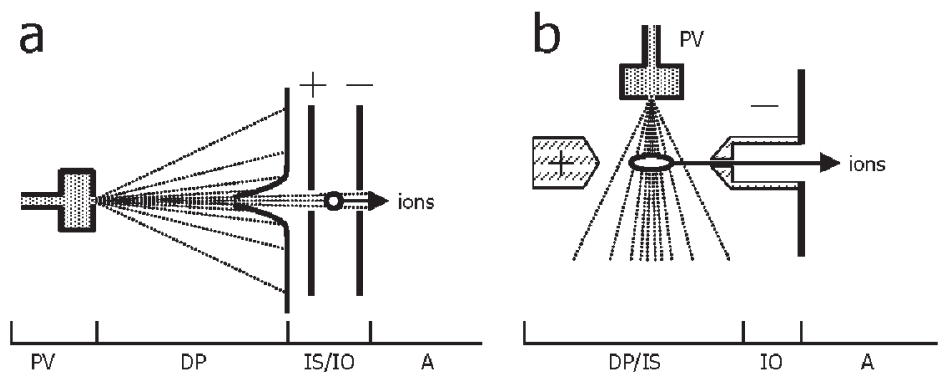

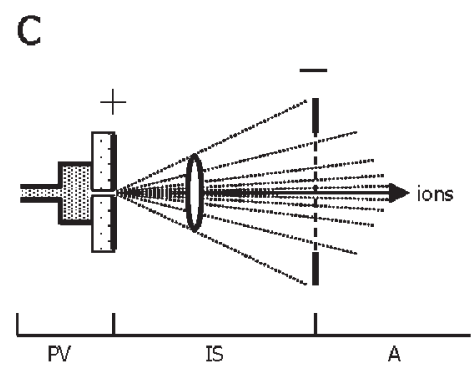

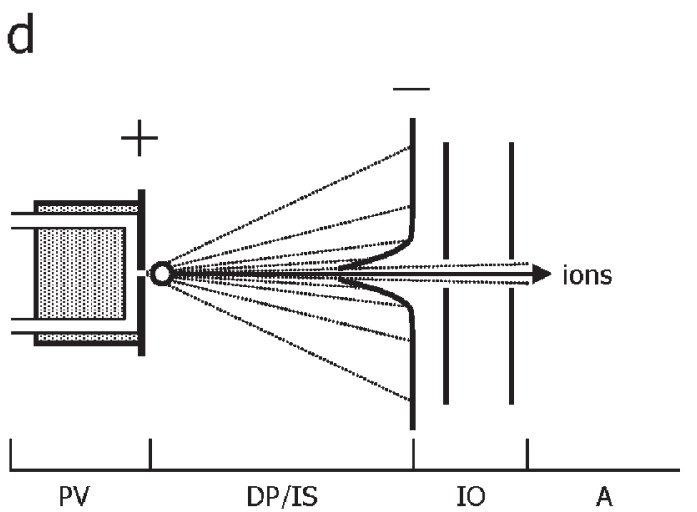

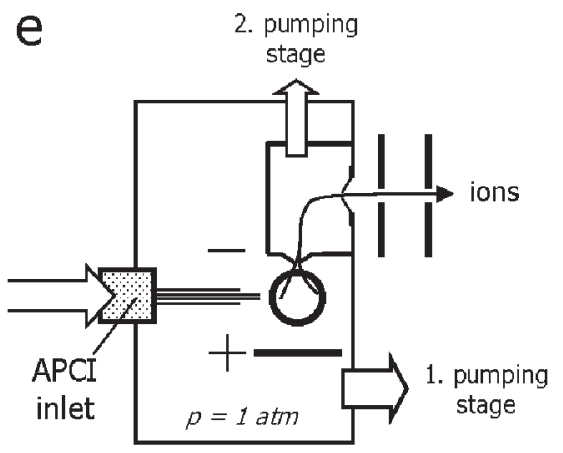

Figure 1. Pulsed inlet systems used in laser ionization mass spectrometry. (a) Traditional skimmed supersonic jet set-up shown in collinear configuration; as for example used in the Bruker TOF1 instrument. Typical nozzle/ionization region distances range from 5 to $20 \mathrm{~cm}$. (b) DLR Jet-REMPI approach [9]. The redesigned ion source geometry allows for significant reduction of the nozzle/ skimmer distance in an orthogonal beam geometry. The unskimmed jet is ionized roughly $2.5 \mathrm{~cm}$ downstream of the nozzle orifice. (c) Collinear ionization scheme as reported by Onoda et al. [12]. This method uses a line beam focus configuration for increased ionization volume, which is located roughly $1 \mathrm{~cm}$ downstream of the unskimmed jet. (d) MPLI approach as described in this contribution. Ionization occurs as close as $0.5 \mathrm{~mm}$ from the nozzle orifice. (e) APLI approach as recently introduced by Constapel et al. Ionization occurs at $\mathrm{p}=1 \mathrm{~atm}$. The generated photo ions are directed towards the orifice by an external electrical field and drawn into the differential pumping stage mainly through bath gas collisions. $\mathrm{PV}=$ pulsed valve; $\mathrm{DP}=$ differential pumping stage; IS = ion source; $\mathrm{IO}=$ ion optics; A = analyzer; \pm high voltage repellor/extraction electrode potentials.

A compact, 40 electrode gridless reflectron was set $2^{\circ}$ off-axis to reflect the ions coaxially to a center-holed, cascaded multi-channel plate assembly (Hamamatsu F2223-21SH, Hamamatsu, Herrsching, Germany) mounted directly onto the ion source exit plate. This system was designed and constructed based on extensive ion trajectory calculations described in detail elsewhere [13]. Data acquisition was accomplished with a 100 ps time-to-digital converter (Ortec 9353, Ortec, Meerbusch, Germany), a pulse delay generator (National Instruments BNC-2121, National Institutes, Austin, TX) and data acquisition electronics (National Instruments DAQ-6602), all mounted into a Pentium PC running LabView in a Windows XP programming environment.

\section{Laser Systems}

For stationary operation, laser ionization was performed with an excimer (Lambda Physik EMG 150,
Lambda Physik, Fort Lauderdale, FL) pumped dye laser (Lambda Physik FL 3002) combination equipped with a second harmonic generation stage. Alteration of the excimer laser hardware allowed the oscillator and amplifier tubes to be operated as independent light sources. One of the tubes pumped the dye laser while the other was typically used to drive the ionization step in multi-color excitation schemes. Both excimer tubes were operated at $308 \mathrm{~nm}\left(\mathrm{XeCl}^{*}\right)$. Tunable UV light $<330 \mathrm{~nm}$ was generated with $\beta$-barium borate (BBO) or potassium titanyl phosphate (KTP) frequency doubling crystals. Maximum pulse energies employed were $10 \mathrm{~mJ}$ for $308 \mathrm{~nm}$ (ionizing beam) and the tunable visible light, and up to $1 \mathrm{~mJ}$ for the second harmonic range. Typical pulse durations, observed at $50 \%$ intensity, are $\sim 11$ ns for the tunable radiation and $\sim 25 \mathrm{~ns}$ for the radiation emitted directly from the excimer laser. Pump and ionization laser pulses were synchronized with a four channel Stanford DG 505 (Stanford, Sunnyvale, CA) 
delay generator. The jitter of the relative arrival time of the two laser light pulses did not exceed $8 \mathrm{~ns}$ as measured with a ThorLabs 201 (Thorlabs, Newton, NJ) fast photodiode detector.

The mobile mass spectrometer was equipped with a software controlled automated portable Lambda Physik OPTex excimer laser, running at $248 \mathrm{~nm}\left(\mathrm{KrF}^{*}\right)$. The laser was mounted on top of the MS frame in addition to beam steering optics. The maximum output energy was $20 \mathrm{~mJ}$, but typical output for experimentation was $5 \mathrm{~mJ}$. The beam was mildly focused using a $30 \mathrm{~cm}$ fused silica lens. Estimated power densities were in the range of $10^{6}-10^{8} \mathrm{~W} / \mathrm{cm}^{2}$ when irradiating the expansion region roughly $5 \mathrm{~cm}$ out of focus at $248 \mathrm{~nm}$.

\section{Pulsed Inlet System}

The pulsed valve is located within a dual chambered glass fiber reinforced Teflon body. The encasing consists of a Teflon membrane sealed solenoid, a Viton tipped stainless steel plunger, two $6 \mathrm{~mm}$ diameter inlet and outlet ports, and a stainless steel faceplate. A $200 \mu \mathrm{m}$ orifice acts as the nozzle. Towards the highpressure side, the plate is equipped with a ceramic inlay, which ensures electrical isolation, and more importantly prevents the gas from being exposed to metal surfaces. The plunger is coated with a Teflon film (Weicon Teflon Spray, Weidling and Sohn, Muenster, Germany), which was sprayed onto the surface and annealed at $80^{\circ} \mathrm{C}$ for $2 \mathrm{~h}$. In separate experiments it was shown that stainless steel surfaces prepared in this manner showed no reactivity toward reactive species such as molecular chlorine or fluorine, gaseous nitric acid, or hydrochloric acid.

Under typical conditions, premixed standard gases, or air directly sampled at atmospheric pressure, was flown through the valve. The analyte mixing ratios and total gas flow were controlled with an MKS up/downstream flow control system as has been shown elsewhere [3].

\section{Ion Source}

The MPLI source is located in the differential pumping chamber of the mass spectrometer and consists of the valve faceplate, a ring electrode supported by two ceramic struts and an electrically isolated skimmer that is mounted on the entrance to the Bruker TOF1 and TurboTOF instruments. Of the ion optics available in the factory equipped mass spectrometers, only the Einzel-lens and the $\mathrm{x}_{-}, \mathrm{y}$-deflection plate potentials of the original ion source were operative. All other electrodes were connected to ground. Figure 2 depicts this set-up. Under typical conditions the potential of the faceplate and ring electrode is held at $\sim 700 \mathrm{~V}$, and the skimmer is kept at ground potential. In the Results section further details are given and different configurations are discussed.

The mobile instrument is equipped with an identical
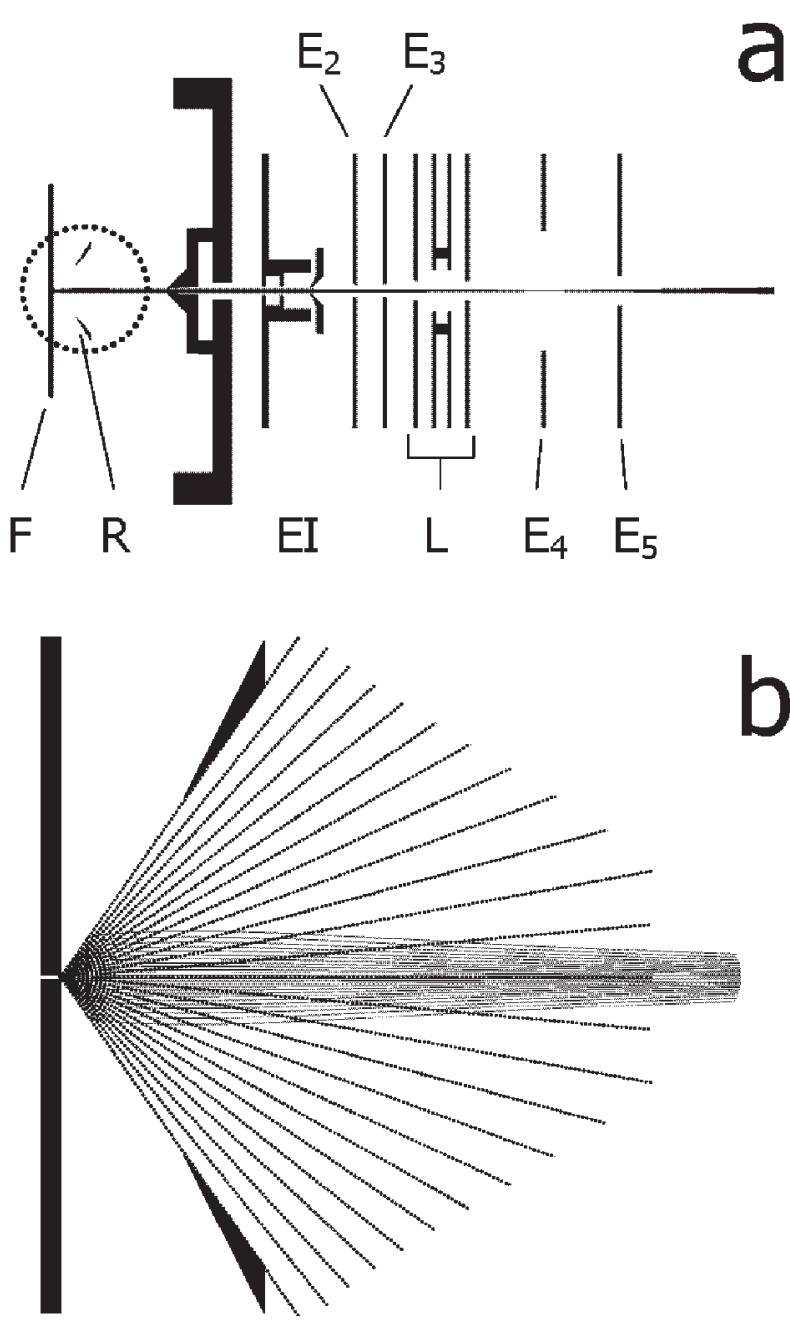

Figure 2. (a) Schematic diagram of the MPLI stage with valve face plate $(\mathrm{F})$, ring electrode $(\mathrm{R})$ and the original Bruker TOF1 ion source comprised of a pulsed electron impact stage (EI), dual acceleration stage $\mathrm{E}_{2}, \mathrm{E}_{3}$, Einzel lens L, and beam steering stage $\mathrm{E}_{4}$, $E_{5}$. All potentials of the original ion source except $L$ are set to zero when running in MPLI mode. (b) Current faceplate/repellor electrode configuration. The cylindrical extension has been replaced by a conical ring electrode for deflection of neutrals and ion extraction/focusing purposes. This set-up minimizes light reflection and thus generation of photoelectrons, which otherwise induce electron impact ionization. In addition, an increased effective ionization volume can be used. Trajectories of neutrals (dotted lines) and ions (solid lines) calculated with the SIMION software package are shown. In this simulation, ionization occurs in the plane indicated by the vertical solid line, $1 \mathrm{~mm}$ downstream of the nozzle orifice.

pulsed valve as described above. The ion optics consist of the valve faceplate, a tube lens mounted in the differential pumping chamber (replacing the ring electrode described above), an Einzel lens and two pairs of ion beam steering plates offset by $90^{\circ}$.

\section{Chemicals}

The carrier and dilution gases helium 5.0 and ultrapure synthetic air were obtained from Oxygen Ser- 
vice Co. (St. Paul, MN) and used without further purification. Premixed NO/He gas (1.1 ppmV) was obtained from Air Gas (Santa Ana, CA) and used directly. Aqueous solutions of $\mathrm{HOCl}(\sim 0.1 \mathrm{~mol} / \mathrm{l})$ were prepared as described in Caldwell et al. [14]. Gaseous $\mathrm{HOCl}$ samples were obtained by bubbling He through a perforator containing the solution and collecting the effluent in a $30 \mathrm{~L}$ Teflon bag. Certified nitrogen dioxide $\left(\mathrm{NO}_{2}\right)$, balanced with $\mathrm{N}_{2}$, was purchased from AGSG with a concentration of 1800 ppmV. Both $\mathrm{NO}$ and $\mathrm{NO}_{2}$ were further purified by three pump-freeze cycles using dry ice baths. A white solid resulted, which was then evaporated and balanced with synthetic air to 900 mbar. A mixture of $10 \%$ ozone $\left(\mathrm{O}_{3}\right)$ in $\mathrm{O}_{2}$ was then added to further remove NO. Benzene, balanced with synthetic air, was purchased from Scott Gas (San Bernadino, CA) with a factory-diluted concentration of $1.08 \mathrm{ppmV}$.

\section{Results and Discussion}

\section{Characterization of the Inlet Valve}

Due to the Teflon construction and ceramic coating of the faceplate, the reactivity of the entire pulsed valve towards the sample is drastically lowered compared to an all-metal design. Furthermore, the dual ported construction with a minimum pumping cross section of $\sim 0.3 \mathrm{~cm}^{2}$ allows the sample gas to flow continuously through the valve. The two $6 \mathrm{~mm}$ bores in the Teflon body are used as inlet and outlet ports, allowing gas flows as high as $100 \mathrm{~L} \mathrm{bar} \mathrm{hr}^{-1}$. Under these conditions, a minimum residence time of the analyte within the valve on the order of a few milliseconds is achieved. As a result, wall loss reactions of reactive species are strongly suppressed, as has been reported in experiments with $\mathrm{HOCl}[14,15]$. In these experiments, the parent ion signal of $\mathrm{HO}^{35} \mathrm{Cl}$ as well as the major product of its heterogeneous decomposition processes, ${ }^{35} \mathrm{Cl}_{2}$, were monitored using electron impact ionization at 52 and $70 \mathrm{Da}$, respectively. The $\mathrm{Cl}_{2}^{+}$ion signal decreased below detection limit at flow rates $>20 \mathrm{~L} \mathrm{bar}^{-1}$ whereas the $\mathrm{HOCl}^{+}$signal reached a plateau.

With the valve operating, the ion source chamber is generally maintained at an average background pressure of $<5 \times 10^{-5}$ mbar. Depending on the electrical driving pulse length (see below), the valve can be pulsed with a frequency up to $100 \mathrm{~Hz}$ when sampling directly from atmospheric pressure. Nonetheless, a more typical operating frequency is between 2 and $20 \mathrm{~Hz}$, matching the repetition rate of the laser system used. In the closed position, the plunger seals the orifice in the faceplate and the sample is continuously flown through the pulsed system via a throttle valve to the pump. The analyzer background pressure drops well below $10^{-7}$ mbar under these conditions.

The solenoid is operated with $100 \mathrm{~V}$ DC pulses of variable length. After the initial phase of the electrical pulse, the valve exhibits a series of open-close cycles on

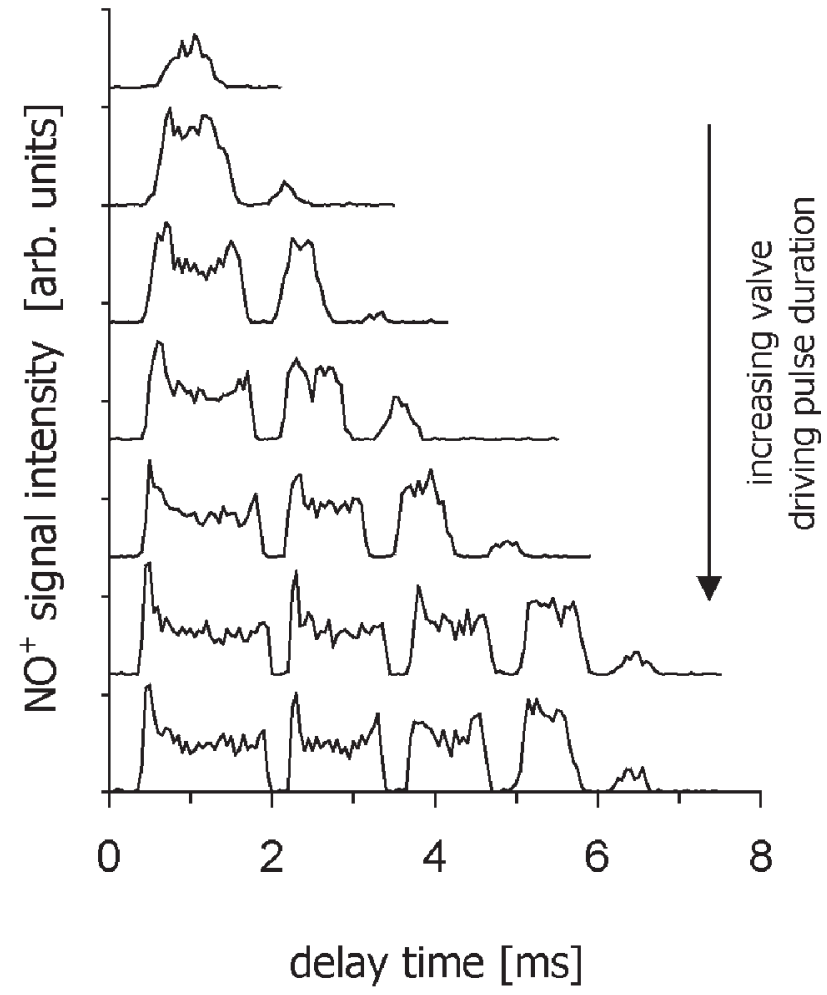

Figure 3. Transient signals of $m / z=30\left(\mathrm{NO}^{+}\right)$recorded as function of the duration of the electrical valve driving pulse. Each scan is generated by stepwise increasing the delay time of the ionization laser pulse relative to the trigger pulse of the valve. From top to bottom the duration of the $100 \mathrm{~V}$ DC valve driving pulse is increased.

its own (ringing). These oscillations are due to the tension in the spring and the Teflon membrane that separates the solenoid and sampling chamber. However, the membrane is essential for the operation of the valve in so far as contamination from the solenoid chamber interferes with NO measurements at ultra-low levels, i.e., $<10 \mathrm{pptV}$. Furthermore, the possibility of analyte loss through wall reactions is greatly reduced. It is thus possible to recycle the gas sampled by flowing it back to the reaction chamber without significantly changing its composition, provided a suitable pumping stage is available (e.g., Teflon membrane pump operated at elevated pressure). By reducing the electrical driving pulse duration, the number of oscillations is reduced. As only one gas pulse is needed for each laser shot, the driving pulse is adjusted to a minimum duration. Figure 3 shows a series of scans generated by delaying the ionizing laser pulse relative to the opening time of the valve. Regardless of the electrical pulse length, the intensity and shape of the initial ion signal remains the same in all but the last two cases. To reduce the amount of gas injected into the ion source, the electrical driving pulse length is set to $\sim 200 \mu \mathrm{s}$.

The sample is irradiated roughly $1 \mathrm{~mm}$ downstream of the nozzle. This point is located in the high-pressure continuous portion of the expanding jet where the density of analyte molecules is still relatively high. 


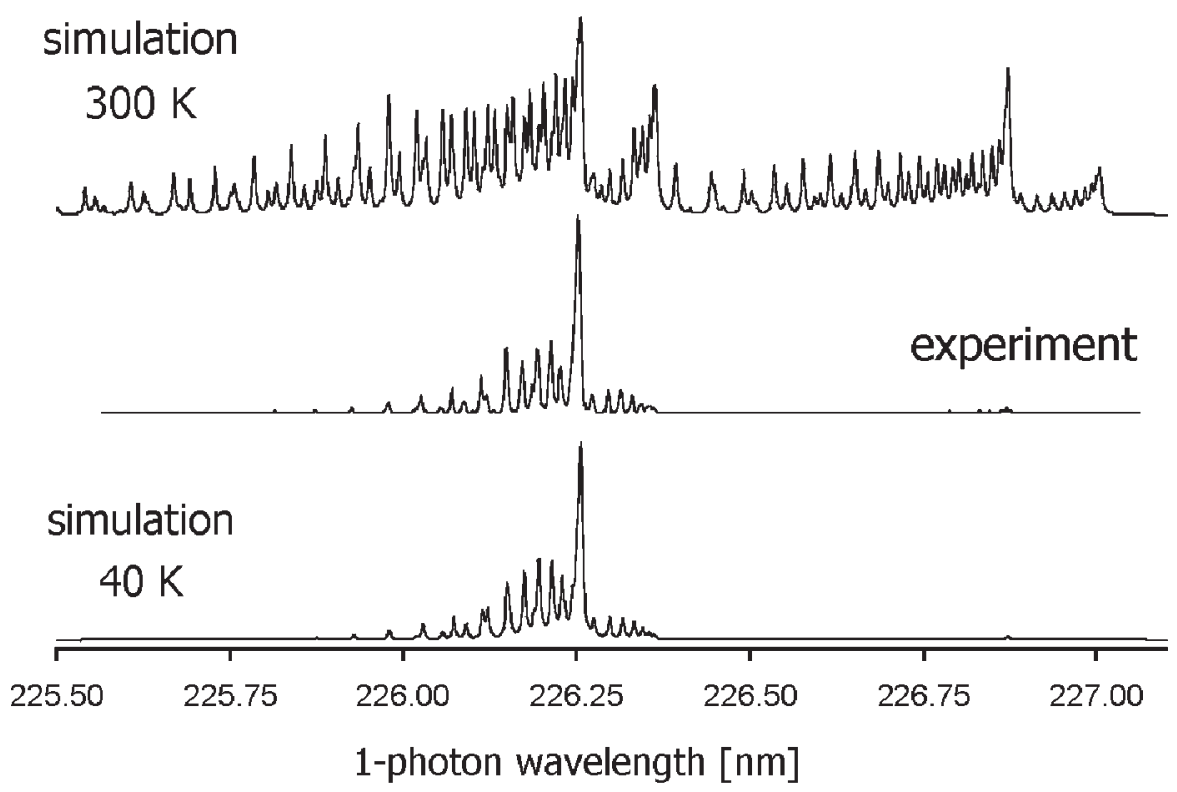

Figure 4. Comparison of simulated NO absorption and $(1+1)$ REMPI spectra. Top and bottom traces: Simulated absorption spectra for the NO A $(\nu=0) \leftarrow \mathrm{X}(\nu=0)$ transition calculated with the LIFBASE program package. Simulation settings: Rovibrational temperature $=300 \mathrm{~K}$ (top trace) and 40 $\mathrm{K}$ (bottom trace), resolution $=0.05 \mathrm{~nm}$, vacuum wavelengths. Middle trace: Experimental $(1+1)$ REMPI spectrum obtained for $\mathrm{NO}^{+}$. The laboratory wavelength scale is shifted to vacuum wavelengths by $0.07 \mathrm{~nm}$.

Further downstream, the trajectories of the neutrals in the evolving jet are parallel to the trajectories of the generated photo ions and therefore the extent of ionmolecule reactions is reduced as compared to a crossbeam set-up. In addition, experimental results show that significant rotational cooling is possible at this short distance from the valve.

Using the thermodynamic approach presented by Miller [7], supersonic jet expansions can be characterized as follows:

$$
\begin{aligned}
& \left(\frac{n_{i}}{n_{o}}\right)^{\gamma-1}=\frac{T_{t, i}}{T_{o}}=\left(1+\frac{\gamma-1}{2} M_{i}^{2}\right)^{-1} \\
& M_{i}=\left(\frac{i}{D}\right)^{(\gamma-11)}\left(3.232-\frac{0.7563}{i / D}+\frac{0.3937}{(i / D)^{2}}-\frac{0.0729}{(i / D)^{3}}\right)
\end{aligned}
$$

Quantities used to characterize expanding jets are usually the Mach number $\left(\mathrm{M}_{\mathrm{i}}\right)$ at a distance $\mathrm{i}$ from the nozzle, the translational temperature $\left(T_{t, i}\right)$ of the gas at a distance i from the nozzle, and the diameter (D) of the nozzle orifice. The Mach number and subsequently the translational temperature is calculated from the preceding two equations, where $T_{o}$ is equal to the stagnation temperature of the gas, $\mathrm{n}_{\mathrm{o}}$ is equal to the stagnation density of the gas, and $n_{i}$ is equal to the gas density at a distance $\mathrm{i}$ from the nozzle. Assuming the jet is composed of a monatomic ideal gas with $\gamma=5 / 3$ for isentropic and continuum flow, we calculate the char- acteristics of the expanding jet produced by the MPLI pulsed valve. Setting $i=1 \mathrm{~mm}$, i.e., the center of the 1 $\mathrm{mm}^{3}$ ionization volume starting $\sim 0.5 \mathrm{~mm}$ off of the face plate, $\mathrm{D}=0.200 \mathrm{~mm}$, and $\mathrm{T}_{\mathrm{o}}=300 \mathrm{~K}$, it is found that $\mathrm{M}_{\mathrm{i}}=9$ and $\mathrm{T}_{\mathrm{t}, \mathrm{i}}=10 \mathrm{~K}$. Although the rotationaltranslational equilibrium is rapidly achieved, the rotational temperature, $T_{r}$, actually achieved is always higher than $T_{t, i}$. From the data given in Miller [7] we calculate a rotational temperature of $\mathrm{T}_{\mathrm{r}}=30 \mathrm{~K}$.

This value is consistent with experimentally recorded data. Figure 4 shows a series of one-photon resonant two-photon ionization $(1+1)$ NO REMPI spectra according to

$$
\begin{aligned}
& \mathrm{NO}(\mathrm{X})+h v \quad(\lambda \approx 226 \mathrm{~nm}) \rightarrow \mathrm{NO}\left[\mathrm{A}^{2} \Sigma^{+}(v=0)\right] \\
& \mathrm{NO}\left[\mathrm{A}^{2} \Sigma^{+}(v=0)\right]+h v \quad(\lambda \approx 226 \mathrm{~nm}) \rightarrow \mathrm{NO}^{+}(\mathrm{X})
\end{aligned}
$$

The top and bottom spectra are generated using the commercially available LIFBASE software package [16] and assume rovibrational temperatures of 300 and $40 \mathrm{~K}$, respectively. The lower trace is an experimental $(1+1)$ REMPI spectrum, which is obtained with the stationary TOF1 set-up. As can been seen, excellent agreement between the experimental and simulated spectra results when a rotational temperature of $40 \mathrm{~K}$ is used for the simulation. We conclude that the MPLI inlet system delivers gas pulses that can be well described by the simple thermodynamic approach and thus reflect the properties of a free jet expansion. 


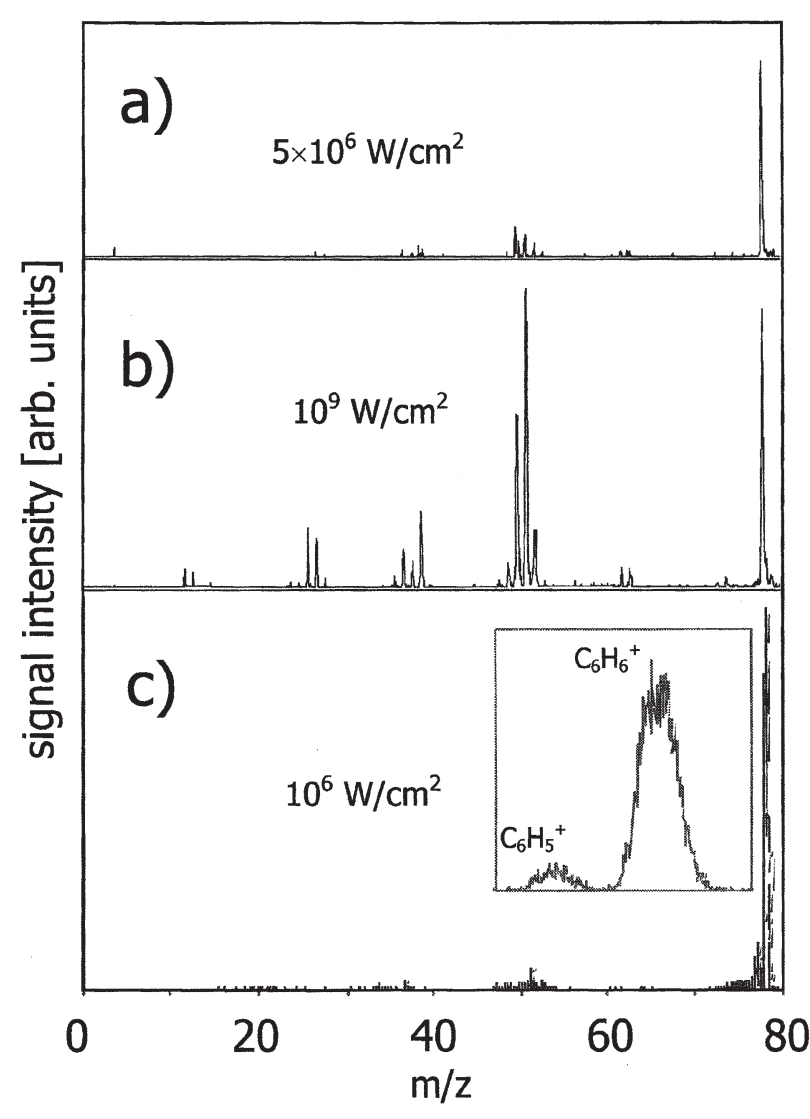

Figure 5. MPLI mass spectra of benzene using $(1+1)$ REMPI with $248 \mathrm{~nm}$ light using the TurboTOF mass spectrometer (a), (b) and the compact system (c). The inset in (c) is an enlarged view of the parent-ion peak showing a mass resolution of $\sim 200(\mathrm{t} / 2 \Delta \mathrm{t})$, calculated by ion trajectory simulations [13]. The approximate laser power density is given on the individual panels.

\section{Characterization of the Ion Source}

Due to the close proximity between the nozzle orifice and the ionization region, the valve faceplate has to be an integral part of the ion source. This incorporation of the pulsed valve into the ion source requires the use of at least two electrodes, i.e., a repellor/extraction electrode combination. The faceplate attached to the valve acts as both an end cap for the valve and as the repellor electrode. It can be biased up to $2 \mathrm{kV}$ but is generally maintained around $700 \mathrm{~V}$. Downstream of the faceplate, a stainless steel skimmer (TOF1 and TurboTOF) or tube lens (home-built TOF) is mounted. Figure $5 \mathrm{a}-\mathrm{c}$ present mass spectra of benzene in air obtained with the MPLI source coupled to the TurboTOF mass spectrometer (Figure 5a, b) and the compact system (Figure 5c).

Unlike skimmed techniques, in the MPLI source the expanding jet is extremely divergent at the point of ionization. The intensity of the expanding jet scales by $\cos (\alpha)^{4}$, where $\alpha$ is the angle with respect to the centerline of the jet [7]. In order to obtain maximum sensitivity, simulations with the SIMION 7.0 [17] software package indicated that an additional electrode was needed to efficiently transfer the ions through the skimmer. The "first generation" MPLI ion source utilized a faceplate equipped with a cylindrical ring attached to the low-pressure side. The cylindrical ring extended $5 \mathrm{~mm}$ off of the faceplate and had an inner diameter of $18 \mathrm{~mm}$. It contained two sets of $5 \mathrm{~mm} \times$ $4 \mathrm{~mm}$ laser beam entrance and exit holes so that one or multiple color REMPI experiments could be performed.

There are a couple of shortcomings associated with this approach for increased sensitivity. First, because the cylinder extends $5 \mathrm{~mm}$ off of the faceplate/repellor electrode, it presents a significant area in which unexcited neutral species can strike. These neutrals then reflect off of the cylinder and possibly participate in reactions by colliding with ions moving towards the detector. Second, and more importantly, the contiguity of the laser beam and the stainless steel surfaces of the cylinder and the faceplate foster light reflection throughout the ion source. Such effects were already noted by Colby and Reilly [18] to be a possibly significant source of interference in laser ionization experiments. It was reported that if the energy of the incoming radiation was larger than the work function of the metal, efficient production of photo electrons was observed. Such electrons will then be immediately accelerated towards the positively biased electrodes and thus through the expanding jet. If the electrons are generated at surfaces held close to ground potential, they will acquire sufficient energy to ionize or fragment virtually any species.

Preliminary experimentation with the first generation repellor electrode design continually resulted in a relatively large $\mathrm{He}^{+}$carrier gas signal relative to the $\mathrm{NO}^{+}$signal of interest. Using the following two-color two-photon process for the detection of $\mathrm{NO}$ in $\mathrm{He}$,

$$
\begin{aligned}
& \mathrm{NO}(\mathrm{X})+h v(\lambda \approx 215 \mathrm{~nm}) \rightarrow \mathrm{NO}\left[\mathrm{A}^{2} \Sigma^{+}(v=0)\right] \\
& \mathrm{NO}\left[\mathrm{A}^{2} \Sigma^{+}(v=0)\right]+h v \quad(\lambda \approx 308 \mathrm{~nm}) \rightarrow \mathrm{NO}^{+}(\mathrm{X})
\end{aligned}
$$

the $\mathrm{He}^{+}$signal completely saturated the detector, therefore severely limiting the attainable sensitivity. Since the ionization potential of $\mathrm{He}$ is $24.6 \mathrm{eV}$ [19], it is apparent that multi-photon ionization cannot be responsible for this signal. A non-resonant 5-photon process is not efficient enough to generate the amount of $\mathrm{He}^{+}$observed. However, due to the low work function of stainless steel of approximately $4.8 \mathrm{eV}$ [20] and the energy of the incoming radiation, i.e., $5.8 \mathrm{eV}(215 \mathrm{~nm})$ and $4.0 \mathrm{eV}(308 \mathrm{~nm})$, efficient production of photoelectrons is easily achieved. It is therefore concluded that the $\mathrm{He}^{+}$signal is the result of ionization via photoelectrons generated by single photon and/or multiphoton processes.

Ion trajectory calculations reveal that the most probable source of photo electrons is due to laser light reflecting off of the cylinder onto the stainless steel skimmer. Experimental evidence supporting the simulations is presented in Figure 6. In these experiments, the skimmer is biased to the same voltage as the 


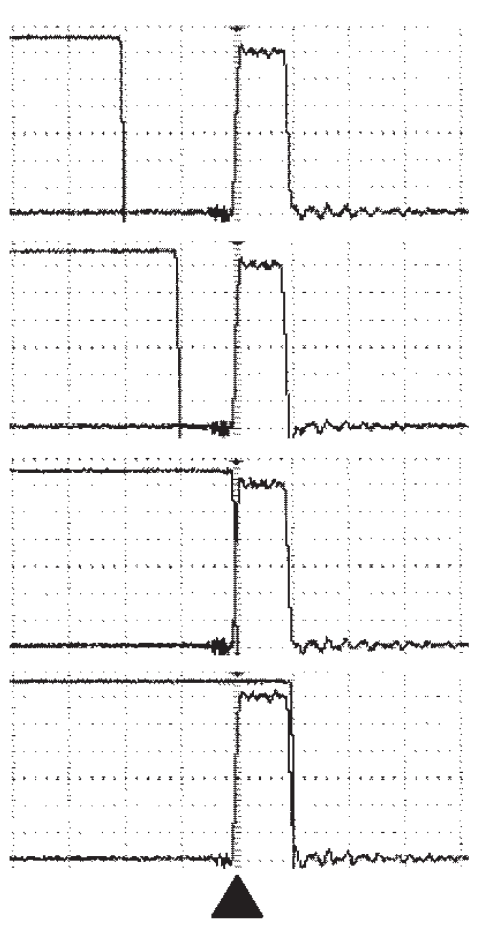

time

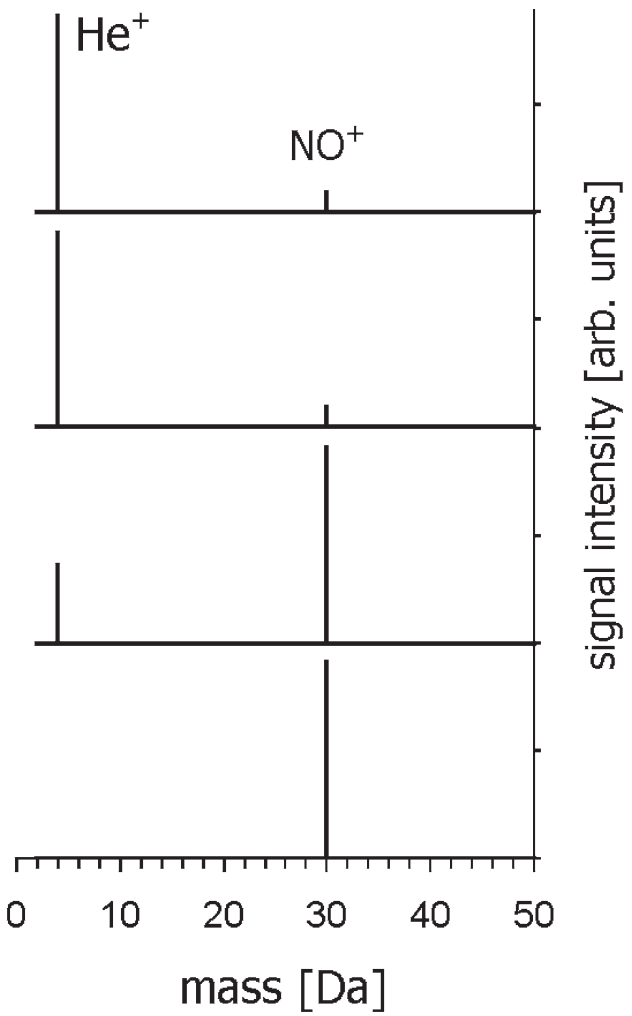

Figure 6. Right panel: Comparison of mass spectra obtained upon biasing the inlet skimmer to the flight tube. Mass spectra recorded with 2-color REMPI $(226 \mathrm{~nm}+308 \mathrm{~nm})$ with a gas flow of $90 \mathrm{ppbV}$ NO in He present. Signal intensities are normalized to the base peak in each spectrum. Notice the absence of $\mathrm{He}^{+}$signals in the bottom mass spectrum where the skimmer is pulsed to ground potential shortly after the light beam arrives in the ion source. Left panel: Digital oscilloscope printouts for the pulses applied to the ionization laser (rectangular pulse starting at $\mathrm{t}=0 \mathrm{~ns}$, indicated by the black arrow) and skimmer voltage driver (only negative slope of pulse visible). Horizontal grid spacing $=$ $250 \mathrm{~ns}$.

repellor electrode and then pulsed to ground at different times relative to the arrival of the laser light. A drastically decreased $\mathrm{He}^{+}$signal is observed when the skimmer is pulsed to ground after the arrival of the laser light. Direct irradiation of the skimmer surface with laser light results in qualitatively identical observations; however, with $\mathrm{He}^{+}$the signal intensities are orders of magnitude higher. It is thus concluded that reflected laser light striking the skimmer surface is the main source of photoelectrons.

Although pulsing the skimmer appears to solve the problem of these rogue electron impact signals, this set-up is still vulnerable to more defocused laser beams. The pulsing experiment was performed while investigating the two-color NO REMPI spectroscopy [2] and required two relatively unfocused laser beams. Further experimentation with $\mathrm{NO}_{2}$ through the following stepwise optical double resonant two-color excitation [3],

$$
\begin{aligned}
& \mathrm{NO}_{2}\left(X^{2} A_{1}\right)+h v(\lambda \approx 431 \mathrm{~nm}) \rightarrow \mathrm{NO}_{2}\left(\mathrm{~A}^{2} \mathrm{~B}_{1}\right) \\
& \mathrm{NO}_{2}\left(\mathrm{~A}^{2} \mathrm{~B}_{1}\right)+h v(\lambda \approx 308 \mathrm{~nm}) \rightarrow \mathrm{NO}_{2}\left(3 p \sigma^{2} \Sigma_{u}^{+}\right) \\
& \mathrm{NO}_{2}\left(3 p \sigma^{2} \Sigma_{u}^{+}\right)+h v(\lambda \approx 308 \mathrm{~nm}) \rightarrow \mathrm{NO}_{2}^{+}(X)
\end{aligned}
$$

which again required two only moderately focused laser beams, led to a significant increase in laser light reflection and the appearance of electron impact signal even under pulsing conditions. As a consequence, a new repellor electrode configuration was designed.

A schematic drawing of the repellor electrode configuration is shown in Figure 3b. A conical ring has replaced the cylinder. This ring is held $8 \mathrm{~mm}$ off of the faceplate by two thin ceramic struts separated by $180^{\circ}$, and is thereby isolated from the potential applied to the faceplate. The inner diameter of the cone at its closest point to the repellor electrode is $18 \mathrm{~mm}$, and if it extended all the way to the faceplate it would make an angle of $50^{\circ}$ relative to a horizontal line extending out from the center of the pulsed valve nozzle. All neutrals that are not ionized and are ejected from the valve with an angle $<50^{\circ}$, travel through the ion source without colliding with a surface until reaching the skimmer, cf. Figure $3 \mathrm{~b}$. Additionally, all neutrals that are emitted from the valve with angles $>50^{\circ}$ will either collide with the conical ring and be reflected away from the expanding jet or will entirely miss the ring. As a result, the new design further reduces interference from ion-molecule reactions.

More importantly, the conical ring design also drastically reduces the amount of laser light reflected 
throughout the ion source. Only the parent ion peak corresponding to $\mathrm{NO}^{+}$is visible in spectra generated with the new ion source, indicating that only a small amount of, if any, electrons are interacting with the expanding jet. The large increase in space in which the laser beam can travel unobstructed is responsible for the lack of electron impact signals.

The ceramic struts isolate the conical ring from the potential of the faceplate and therefore allow the ring to be biased independently from the repellor electrode. Simulations and experimentation reveal that maximum sensitivity is achieved when the ring is biased to potentials slightly less than that of the repellor electrode. Under these conditions the ring behaves as an aperture lens and focusing of ions through the skimmer is achieved. These focusing characteristics, coupled with the location of ionization, allow for the extremely sensitive detection of gas phase compounds. Due to the much smaller dimensions of the differential pumping stage of the home-built instrument and thus less favorable aerodynamic parameters, the face plate-ring-skimmer configuration was replaced with a face plate - tube lens set-up resulting in comparable ion transport efficiencies through the analyzer orifice as shown by ion trajectory calculations.

\section{Sensitivity}

Using eqs $1 \mathrm{a}$ and $1 \mathrm{~b}$, the density of the expanding jet can be calculated along the centerline axis. With an analyte mixing ratio of $1 \mathrm{pptV}$ at atmospheric pressure within the valve, the sample density $1 \mathrm{~mm}$ off the faceplate in the differential pumping stage is predicted to be $\sim 2 \times 10^{5}$ molecules $\mathrm{cm}^{-3}$. Assuming an ionization volume of $1 \mathrm{~mm}^{3}$, a maximum of 200 molecules are available for detection. If ionization occurs $50 \mathrm{~mm}$ off the faceplate, the sample density is roughly 60 molecules $\mathrm{cm}^{-3}$, leaving less than 1 molecule for detection within the ionization volume. Based on these calculations, a theoretical sensitivity enhancement of $\sim 3000$ is gained over traditional techniques.

This sensitivity enhancement has also been verified experimentally $[2,3]$. When coupled to the Bruker TOF1, the MPLI source fosters detection of NO down to sub-pptV levels. In these experiments, the instrument response was monitored as a function of the nitric oxide mixing ratio. Using the ionization scheme outlined in eqs $3 a$ and $3 b$ above, an integration time of 20 seconds, and an ionization volume of less than $1 \mathrm{~mm}^{3}$, a detection limit for $\mathrm{NO}$ of $0.5 \mathrm{pptV}$ is calculated from

$$
C_{1}=\frac{k \sigma_{b l}}{S}
$$

where $\sigma_{b l}$ is the standard deviation of the blank signal, $S$ is the calibration sensitivity, and the constant $k$ is a multiple of the standard deviation and as argued in Skoog et al. [21] is set equal to 3. This is an order of magnitude lower than the value reported by Lee et al.
$[11,22]$ in which an ionization volume of $5000 \mathrm{~mm}^{3}$ and integration time of $1 \mathrm{~min}$ was used.

Additionally, a detection limit of $12 \mathrm{pptV}$ with an integration time of $10 \mathrm{~s}$ for 2,5-DCT has been achieved with MPLI, which is considerably lower than the value of $100 \mathrm{pptV}$ reported by Oser et al. [9]. In their experiments, a $10 \mathrm{~mm}^{3}$ ionization volume and an integration time of 20 seconds was used.

It is pointed out that particulate matter, one of the components of most complex gas phase samples, will have little, if any, effect on the efficacy of the MPLI approach. With the exception of particle obstruction of the nozzle orifice, which will only occur when sampling from the most congested sources, e.g., combustion engines or incineration plants, the two primary impacts that particulate matter may have on the accurate detection of trace gases in complex mixtures are contamination from laser desorption/ionization of analytes from the particle surface and adsorption of analytes within the jet expansion. However, it must be stressed that MPLI is not designed to be used in congested or heavily polluted environments. Rather, MPLI applications are geared towards highly dynamic systems, e.g., reacting chemical systems in atmospheric smog chambers or time-resolved human breath analysis.

For the measurement of exhaled air, only small number densities of particulate matter (i.e., $<10^{3}$ particles $/ \mathrm{cm}^{3}$ ) are expected due to the efficient trapping of aerosols throughout the respiratory tract. In the remote atmosphere typical particle concentrations are approximately $10^{3}$ particles $/ \mathrm{cm}^{3}$ [23]. At this level, far less than one particle on average will reside within the ionization volume per 30 pulses, severely limiting the effect that particles can have on any analytical results. In the urban atmosphere, typical aerosol concentrations reach $10^{5}$ particles $/ \mathrm{cm}^{3}$. At this mixing ratio, it is expected that one particle will be within the ionization volume per 30 laser pulse events. Even at these relatively large particle densities, contamination from laser desorption/ ionization of a few particles will be insignificant. In addition, UV laser desorption processes from surfaces result in relatively large velocity [24] distributions of analyte molecules. Due to the location of the analytes on the spherical particles, the laser-desorbed molecules will also be ejected at a variety of angles relative to the TOF axis. As has been discussed, the MPLI geometry samples predominantly analyte molecules with flight trajectories close to the TOF axis. Thus, further discrimination of laser-desorbed species is expected.

Additionally, adsorption of analyte molecules on particles downstream of the nozzle will not affect the sensitivity of the MPLI technique. Depending on the molecule and the surface composition of the particle, the sticking coefficient (i.e., the probability of a molecule being absorbed on the surface of a particle per collision) may range from 1 to $<10^{-8}$. As an example of the impact this type of contamination will have on our instrument, we will look at the effect that the presence of particles may have on the detection of $\mathrm{NO}_{2}$. Assum- 
ing the particles within the sampled air are all carbon aerosol, the range of relatively large sticking coefficients is reported to extend from $1.1 \times 10^{-2}$ to $2.4 \times 10^{-4}$ [25]. For simplicity, we will assume an average sticking coefficient of $10^{-3}$. In a free jet expansion in which all species are traveling at or near the same velocity, a typical molecule will undergo $10^{2}$ to $10^{3}$ binary collisions [7]. However, due to the restricted velocity of "heavy" atmospheric particles relative to gases seeded in a supersonic jet, this value may underestimate the number of collisions in a jet expansion containing particulate matter. As a result, if we assume all of these collisions happen prior to ionization $1 \mathrm{~mm}$ downstream of the nozzle orifice, and $\mathrm{NO}_{2}$ only collides with particles, then the probability of $\mathrm{NO}_{2}$ sticking to the surface of the particle will be unity. Nonetheless, although the particles provide a larger target for collision and may increase the total number of collisions within the expansion, due to the low particle $\left(\sim 10^{5}\right.$ particles $\left./ \mathrm{cm}^{3}\right)$ and analyte $\left(\sim 10^{12}\right.$ molecules $\left./ \mathrm{cm}^{3}\right)$ concentrations found in urban air, it is still more likely that the analyte will collide with carrier gas (i.e., $\mathrm{N}_{2}$ or $\mathrm{O}_{2}$ ) than with a particle. This is in agreement with Cleary et al., who do not report problems with analyte adsorption when using a supersonic expansion coupled with laser-induced fluorescence to detect atmospheric $\mathrm{NO}_{2}$ [26]. Therefore, we expect minimal particle interference in the analysis and detection of $\mathrm{NO}_{2}$ and other molecules with comparable sticking coefficients.

\section{Comparison of Jet-REMPI, MPLI, and APLI}

Very recently, Constapel et al. [4] have reported on the development of a novel atmospheric pressure laser ionization technique, APLI. As already mentioned in the introduction, this acronym was used before by us for the technique described in detail in this paper. However, in order to properly label the different ionization techniques, we chose MPLI as the more appropriate acronym representing the present laser ionization method.

The three basic approaches for analytical applications of REMPI, namely (1) jet-REMPI, (2) MPLI, and (3) APLI all have advantages and limitations and thus coexist as powerful laser based ionization techniques, which are briefly discussed in the section below.

The jet-REMPI approach is geared toward highly specific detection of rather complex analytes, such as dioxins in flue gases of incineration plants. The emphasis lies apparently on maximum attainable selectivity. Consequently, detection of analytes takes place when maximum rotational cooling is achieved, i.e., downstream of the continuous region of the jet. Jet-REMPI is frequently used in combination with narrow bandwidth $\left(\leq 0.1 \mathrm{~cm}^{-1}\right)$ laser sources such as excimer pumped dye lasers or solid state laser pumped narrow bandwidth OPOs. These arrangements result in rather bulky setups better suited as stationary instruments for the above mentioned applications [27]. Furthermore, pre- cise knowledge of the spectroscopy of the analytes of interest is required in order to be able to exploit the advantages of jet-REMPI.

MPLI aims at analytes in the mass range 10-200 Da. Primary motivation for developing MPLI is to provide a time-resolved laser ionization technique in the second to sub-second range and increasing the sensitivity compared to the traditional jet-REMPI approach. Rotational cooling is far less efficient compared to jet-REMPI; the rotational temperature is on the order of $50 \mathrm{~K}$ within the ionization volume. Thus medium to broad bandwidth light sources $\left(1 \mathrm{~cm}^{-1}<\lambda<50 \mathrm{~cm}^{-1}\right)$ such as compact solid-state laser pumped OPOs are favorably employed. Simultaneous excitation of a number of rotational states compensates for the loss of ionization efficiency when using narrow bandwidth excitation under these conditions. Footprint as well as complexity of the light source is significantly reduced. In this article, we have shown the adaptation of MPLI to a compact TOF-MS. Analyte mass range, bandwidth of the light source, and size of the overall instrument are thus ideally matched for mobile operation. Molecules in the above mentioned mass range frequently exhibit spectroscopic patterns that can be used for unequivocal identification. Either single- or two-color schemes (i.e., excitation/ionization or optical double resonance) lead to high selectivities of the method.

APLI employs laser excitation at atmospheric pressure and ambient temperatures [4]. The technique is geared toward the ultra-sensitive analysis of analytes containing aromatic functions in the mass range $>100$ $\mathrm{Da}$, providing an alternative to atmospheric pressure chemical ionization (APCI) and photoionization (APPI). Since larger molecular systems containing aromatic functions usually exhibit broad and fairly unresolved absorption spectra in the near UV, in particular at room temperature, step-wise two-photon excitation with unfocused fixed frequency lasers (e.g., $\mathrm{N}_{2}, \mathrm{XeCl}^{*}, \mathrm{KrF}^{*}$, and $\mathrm{ArF}^{*}$ lasers) leads to highly efficient parent ion production. Furthermore, this technique is ideally suited to be coupled with preseparation stages, e.g., HPLC. Besides direct generation of the parent ions, a number of new applications of REMPI may arise from here, comparable to, e.g., dopant assisted photoionization (DA APPI) [28], or photoelectron resonance capture ionization (PERCI) [29].

\section{Conclusions}

The MPLI pulsed valve/ion source combination is ideally suited to time of flight mass spectrometry. Due to the extremely small nozzle-laser beam distance, MPLI offers a significant increase in sensitivity relative to current laser ionization mass analysis techniques. The temporal resolution is determined basically by the repetition rate and the desired sensitivity and approaches the sub-second range for $\mathrm{ppbV}$ mixing ratios. The inert construction and large flow velocity of the pulsed valve allow for sampling of reactive species, and 
despite the short distance to the location of ionization, significant rotational cooling is achieved. Furthermore, the adaptation of a conical ring or a tube lens to the MPLI ion source has virtually eliminated the generation of photoelectrons by drastically reducing electron impact signals.

The MPLI source was coupled to portable broadand narrow-bandwidth laser/TOF-MS systems as well as stationary systems. Results reported in a recent paper are promising [30]. It is shown that under limited rotational cooling conditions the efficiency of selected photo ionization processes in conjunction with broad-bandwidth laser excitation results in a similar performance with respect to sensitivity compared to the jet-cooling/narrow bandwidth excitation approach.

As a result of its ability to sample directly from atmospheric pressure, its high sensitivity, and its real-time capabilities, MPLI is well suited to a portable instrument and might find use in a variety of fields. Future applications will include environmental monitoring of atmospherically relevant species such as $\mathrm{NO}, \mathrm{NO}_{2}$, formaldehyde, aromatic hydrocarbons, and serving the medical field as a non-invasive diagnostic tool by measuring trace species such as $\mathrm{NO}$, acetaldehyde, acetone, $\mathrm{NH}_{3}$, etc. that are found in human breath.

\section{Acknowledgments}

This work was supported by the NSF Atmospheric Program, grant no. 0083436 , and in part by the BMBF, Germany, within projects 01 LO 950/6 and 07 AK 302/0. LCS acknowledges support through a fellowship of the Fulbright Foundation.

\section{References}

1. Koenig, J. Q. Health Effects of Ambient Air Pollution: How Safe Is The Air We Breathe? Kluwer Academic: Boston, 2000; p 249.

2. Schmidt, S.; Appel, M. F.; Garnica, R. M.; Schindler, R. N.; Benter, T. Atmospheric Pressure Laser Ionization. An Analytical Technique for Highly Selective Detection of Ultralow Concentrations in the Gas Phase. Anal. Chem. 1999, 71, 37213729.

3. Garnica, R. M.; Appel, M. F.; Eagan, L.; McKeachie, J. R.; Benter, T. A REMPI Method for the Ultrasensitive Detection of $\mathrm{NO}$ and $\mathrm{NO}_{2}$ Using Atmospheric Pressure Laser Ionization Mass Spectrometry. Anal. Chem. 2000, 72, 5639-5646.

4. Constapel, M.; Schellentraeger, M.; Moeschter, S.; Schmitz, O.; Gaeb, S.; Brockmann, K.; Giese, R.; Benter, T. Introduction of a New Atmospheric Pressure Laser Ionization (APLI) Source for LC MS and in Situ Gas Phase Sampling Applications. Proceedings of the 53rd Meeting of the ASMS Conference; Nashville, TN, May 2004, pp 2624-2626.

5. Scoles, G. In Atomic and Molecular Beam Methods; Laine, D.; Valbusa, U., Eds.; Oxford University Press: New York, 1992; p 552.

6. Boesl, U.; Weinkauf, R.; Weickhardt, C.; Schlag, E. W. Laser Ion Sources For Time-of-Flight Mass Spectrometry. Int. J. Mass Spectrom. Ion Processes 1994, 131, 87-124.

7. Miller, D.R. Free Jet Sources. In Atomic and Molecular Beam Methods; Scoles, G., Ed.; Oxford University Press: New York, 1988; p 721.
8. Oser, H.; Coggiola, M. J.; Faris, G. W.; Young, S. E.; Volquardsen, B.; Crosley, D. R. Development of a Jet-REMPI (Resonantly Enhanced Multiphoton Ionization) Continuous Monitor for Environmental Applications. Appl. Opt. 2001, 40, 859865 .

9. Oser, H.; Thanner, R.; Grotheer, H. H. Jet-REMPI for the Detection of Trace Gas Compounds in Complex Gas Mixtures, a Tool for Kinetic Research and Incinerator Process Control. Combust. Sci. Technol. 1996, 116, 567-582.

10. Castaldi, M. J.; Senkan, S. M. Real-Time, Ultrasensitive Monitoring of Air Toxics by Laser Photoionization Time-of-Flight Mass Spectrometry. J. Air Waste Manag. Assoc. 1998, 48, 77-81.

11. Lee, S. H.; Kajii, Y.; Akimoto, H. Improvement of the New NO Detection Method Using Laser-Induced Two-Photon Ionization with a TOF Mass Spectrometer. Environ. Sci. Technol. 2000, 34, 4434-4438.

12. Onoda, T.; Saito, G.; Imasaka, T. Scheme for Ccollinear Ionization in Supersonic Jet/Multiphoton Ionization/Time-ofFlight Mass Spectrometry. Anal. Chim. Acta 2000, 412, 213219.

13. Appel, M. The Devolpment of Portable Instrumentation for Laser Ionization Time-of-Flight Mass Spectrometry. Ph.D. Thesis, University of California, Irvine, 2003.

14. Caldwell, T. E.; Foster, K. L.; Benter, T.; Langer, S.; Hemminger, J. C.; Finlayson-Pitts, B. J. Characterization of HOCL Using Atmospheric Pressure Ionization Mass Spectrometry. J. Phys. Chem. A 1999, 103, 81-8238.

15. Wayne, R. P.; Poulet, G.; Biggs, P.; Burrows, J. P.; Cox, R. A.; Crutzen, P. J.; Hayman, G. D.; Jenkin, M. E.; Lebras, G.; Moortgat, G. K.; Platt, U.; Schindler, R. N. Halogen Oxides-Radicals, Sources, and Reservoirs in the Laboratory and in the Atmosphere. Atmosph. Environ. 1995, 29, 26772881.

16. Luque, J.; Crosley, D. R. LIFBASE: Database and Spectral Simulation Program, Version 1.5; SRI International Report MP 99009:

17. Dahl, D. A. Simion 7.0; Ion Source Software: ID 83403; Idaho Falls, ID 83403.

18. Colby, S. M.; Reilly, J. P. Photoemission Electron Impact Ionization in Time-of-Flight Mass Spectrometry-An Examination of Experimental Consequences. Int. J. Mass Spectrom. Ion Processes 1994, 131, 125-138.

19. Rosenstock, H. M.; Draxl, K.; Steiner, B. W.; Herron, J. T. Energetics of Gaseous Ions. J. Phys. Chem. Ref. Data 1977, 6, 70.

20. Walton, S. G.; Champion, R. L.; Wang, Y. Negative Ion Emission from a Stainless Steel Surface Due to Positive Ion Collisions. J. Appl. Phys. 1998, 84, 1706-1707.

21. Skoog, D. A; Holler, F. J; Nieman, T. A. Principles of Instrumental Analysis; Saunders College Publication: Philadelphia, 1998; p 849

22. Lee, S. H.; Hirokawa, J.; Kajii, Y.; Akimoto, H. New Method for Measuring Low NO Concentrations Using Laser Induced Two Photon Ionization. Rev. Sci. Instr. 1997, 68, 2891-2897.

23. Finlayson-Pitts, B. J; Pitts, J. N. Chemistry of the Upper and Lower Atmosphere: Theory, Experiments, and Applications; Academic Press: San Diego, 2000; p 969

24. Amoruso, S.; Berardi, V.; Bruzzese, R.; Spinelli, N.; Wang, X. Kinetic Energy Distributions of Ions in the Laser Ablation of Copper Targets. 1998,127, 953-958.

25. Wayne, R. P.; Poulet, G.; Biggs, P.; Burrows, J. P.; Cox, R. A.; Crutzen, P. J.; Hayman, G. D.; Jenkin, M. E.; Le Bras, G.; Moortgat, G. K.; Platt, U.; Schindler, R. N. Halogen Oxides: Radicals, Sources, and Reservoirs in the Laboratory and in the Atmosphere. Atmosph. Environ. 1995, 29, 26772881. 
26. Cleary, P. A.; Wooldridge, P. J.; Cohen, R. C. Laser-Induced Fluorescence Detection of Atmospheric $\mathrm{NO}_{2}$ with a Commercial Diode Laser and a Supersonc Expansion. Appl. Opt. 2002, 41, 6950-6956.

27. Oser, H.; Thanner, R.; Grotheer, H.; Gullett, B.; Bergan French, N.; Natschke, D. DLR Jet-REMPI as a Continuous Emissions Monitor: Measurements of Chlorinated Dibenzodioxins. Proceedings of the International Conference on Incineration and Thermal Treatment Technologies; Oakland, CA, May 1997, pp 12-16.

28. Robb, D. B.; Covey, T. R.; Bruins, A. P. Atmospheric Pressure
Photoionisation: An Ionization Method for Liquid Chromatography-Mass Spectrometry. Anal. Chem. 2000, 72, 3653-3659.

29. LaFranchi, B. W.; Petrucci, G. A. Photoelectron Resonance Capture Ionization (PERCI): A Novel Technique for the SoftIonization of Organic Compounds. J. Am. Soc. Mass Spectrom. 2004, 15, 424-430.

30. McKeachie, J. R.; Van der Veer, W. E.; Short, L. C.; Garnica, R. M.; Appel, M. F.; Benter, T. Selective Ultra-Trace Detection of NO and $\mathrm{NO}_{2}$ in Complex Gas Mixtures Using Broad-Bandwidth REMPI Mass Spectrometry. Analyst 2001, 126, 1221-1228. 